\title{
ANNIHILATOR OF LOCAL COHOMOLOGY MODULES AND STRUCTURE OF RINGS
}

\author{
Tran Nguyen An \\ TNU - University of Education
}

\section{ABSTRACT}

Let $(R, \mathrm{~m})$ be a Noetherian local ring, $A$ an Artinian $R$-module, and $M$ a finitely generated $R$ module. It is clear that $\operatorname{Ann} R(M / \mathrm{p} M)=\mathrm{p}$, for all $\mathrm{p} \in \operatorname{Var}(\operatorname{Ann} R M)$. Therefore, it is natural to consider the following dual property for annihilator of Artinian modules:

$$
\text { Ann } R(0: A \mathrm{p})=\mathrm{p} \text {, for all } \mathrm{p} \in \operatorname{Var}(\operatorname{Ann} R A) \text {. (*) }
$$

Let $i \geq 0$ be an integer. Alexander Grothendieck showed that the local cohomology module $H \mathrm{~m} i$ $(M)$ of $M$ is Artinian. The property (*) of local cohomology modules is closed related to the structure of the base ring. In this paper, we prove that for each $\mathrm{p} \in \operatorname{Spec}(R)$ such that $H \mathrm{~m} i(R / \mathrm{p})$ satisfies the property (*) for all $i$, then $R / \mathrm{p}$ is universally catenary and the formal fibre of $R$ over $\mathrm{p}$ is Cohen-Macaulay.

Keywords: Local cohomology; universally catenary; formal fibre; Artinian module; CohenMacaulay ring

Received: 26/5/2020; Revised: 29/8/2020; Published: 04/9/2020

\section{LINH HÓA TỦ CỦA MÔĐUN ĐỐI ĐỒNG ĐIỀU ĐỊA PHỦƠNG VÀ CẤU TRÚC VÀNH}

Trần Nguyên An

Trưòng Đại học Su phạm - ĐH Thái Nguyên

\section{TÓM TẮT}

Cho $(R, \mathrm{~m})$ là vành Noether địa phương, $A$ là $R$-môđun Artin, và $M$ là $R$-môđun hữu hạn sinh. Ta có Ann $R(M / \mathrm{p} M)=\mathrm{p}$ với mọi $\mathrm{p} \in \operatorname{Var}(\operatorname{Ann} R M)$. Do đó rất tự nhiên ta xét tính chất sau về linh hóa tử của môđun Artin

Ann $R(0: A \mathrm{p})=\mathrm{p}$ for all $\mathrm{p} \in \operatorname{Var}(\operatorname{Ann} R A)$. (*)

Cho $i \geq 0$ là số nguyên. Alexander Grothendieck đã chỉ ra rằng môđun đối đồng điều địa phương $H i \mathrm{~m}(M)$ là Artin. Tính chất $(*)$ của các môđun đối đồng điều địa phương liên hệ mật thiết với cấu trúc vành cơ sở. Trong bài báo này, chúng tôi chỉ ra với mỗi $\mathrm{p} \in \operatorname{Spec}(R)$ mà $H \mathrm{~m} i(R / \mathrm{p})$ thỏa mãn tính chất $(*)$ với mọi $i$ thì $R / \mathrm{p}$ là catenary phổ dụng và các thớ hình thức của $R$ trên $\mathrm{p}$ là CohenMacaulay.

Từ khóa: Đối đồng điều địa phuoong; catenary phổ dụng; thó hình thức; môđun Artin; vành Cohen-Macaulay

Ngày nhận bài: 26/5/2020; Ngày hoàn thiện: 29/8/2020; Ngày đăng: 04/9/2020 


\section{Introduction}

Throughout this paper, let $(R, \mathfrak{m})$ be a Noetherian local ring, $A$ an Artinian $R$ module, and $M$ a finitely generated $R$ module of dimension $d$. For each ideal $I$ of $R$, we denote by $\operatorname{Var}(I)$ the set of all prime ideals containing $I$. For a subset $T$ of $\operatorname{Spec}(R)$, we denote by $\min (T)$ the set of all minimal elements of $T$ under the inclusion.

It is clear that $\operatorname{Ann}_{R}(M / \mathfrak{p} M)=\mathfrak{p}$, for all $\mathfrak{p} \in \operatorname{Var}\left(\operatorname{Ann}_{R} M\right)$. Therefore, it is natural to consider the following dual property for annihilator of Artinian modules:

$$
A n n_{R}\left(0:_{A} \mathfrak{p}\right)=\mathfrak{p}, \forall \mathfrak{p} \in \operatorname{Var}\left(\operatorname{Ann}_{R} A\right) .(*)
$$

If $R$ is complete with respect to $\mathfrak{m}$-adic topology, it follows by Matlis duality that the property $(*)$ is satisfied for all Artinian $R$ modules. However, there are Artinian modules which do not satisfy this property. For example, by [1, Example 4.4], the Artinian $R$-module $H_{\mathfrak{m}}^{1}(R)$ does not satisfy the prop$\operatorname{erty}(*)$, where $R$ is the Noetherian local domain of dimension 2 constructed by M. Ferrand and D. Raynaud [2] (see also [3, App. Ex. 2] Ex. 2]) such that its m-adic completion $\widehat{R}$ has an associated prime $\mathfrak{q}$ of dimension 1. In [4], N. T. Cuong, L. T. Nhan and N. T. Dung showed that the top local cohomology module $H_{\mathfrak{m}}^{d}(M)$ satisfies property $(*)$ if and only if the ring $R / \operatorname{Ann}_{R}\left(M / U_{M}(0)\right)$ is catenary, where $U_{M}(0)$ is the largest submodule of $M$ of dimension less than $d$. The property $(*)$ of local cohomology modules is closed related to the structure of the ring. In [5], L. T. Nhan and the author proved that if $H_{\mathfrak{m}}^{i}(M)$ satisfies the property $\left(^{*}\right)$ for all $i$, then $R / \mathfrak{p}$ is unmixed for all $\mathfrak{p} \in \operatorname{Ass} M$ and the ring $R / \operatorname{Ann}_{R} M$ is universally catenary. The following conjecture was given by N. T. Cuong in his seminar.

Conjecture 1.1. The following statements are equivalent: (i) $H_{\mathfrak{m}}^{i}(R)$ satisfies the property $\left(^{*}\right)$ for all $i$;

(ii) $R$ is universally catenary and all its formal flbers are Cohen-Macaulay.

L. T. Nhan and T. D. M. Chau proved in [6] that $H_{\mathfrak{m}}^{i}(M)$ satisfies the property $\left({ }^{*}\right)$ for all $i$, for all finitely generated $R$-module $M$ if and only if $R$ is universally catenary and all its formal flbers are Cohen-Macaulay. The following result is the main result of this paper. We hope that we can use this to give a positive answer for the above conjecture.

Theorem 1.2. Assume $\mathfrak{p} \in \operatorname{Spec}(R)$ such that $H_{\mathfrak{m}}^{i}(R / \mathfrak{p})$ satisfies the property $\left(^{*}\right)$ for all $i$. Then $R / \mathfrak{p}$ is universally catenary and the formal fibre of $R$ over $\mathfrak{p}$ is CohenMacaulay.

\section{Proof of the main results}

The theory of secondary representation was introduced by I. G. Macdonald (see [7]) which is in some sense dual to that of primary decomposition for Noetherian modules. Note that every Artinian $R$-module $A$ has a minimal secondary representation $A=A_{1}+\ldots+A_{n}$, where $A_{i}$ is $\mathfrak{p}_{i}$-secondary. The set $\left\{\mathfrak{p}_{1}, \ldots, \mathfrak{p}_{n}\right\}$ is independent of the choice of the minimal secondary representation of $A$. This set is called the set of attached prime ideals of $A$, and denoted by $\operatorname{Att}_{R} A$. Note also that $A$ has a natural structure as an $\widehat{\mathrm{R}}$-module. With this structure, a subset of $A$ is an $R$-submodule if and only if it is an $\widehat{\mathrm{R}}$-submodule of $A$. Therefore, $A$ is an $\mathrm{Ar}$ tinian $\widehat{\mathrm{R}}$-module.

Lemma 2.1. (i) The set of all minimal elements of $\operatorname{Att}_{R} A$ is exactly the set of all minimal elements of $\operatorname{Var}\left(\operatorname{Ann}_{R} A\right)$.

(ii) $\operatorname{Att}_{R} A=\left\{\widehat{\mathfrak{p}} \cap R: \widehat{\mathfrak{p}} \in \operatorname{Att}_{\widehat{\mathrm{R}}} A\right\}$. 
R. N. Roberts introduced the concept of Krull dimension for Artinian modules (see [8]). D. Kirby changed the terminology of Roberts and referred to Noetherian dimension to avoid confusion with Krull dimension defined for finitely generated modules (see [9]). The Noetherian dimension of $A$ is denoted by $\mathrm{N}-\operatorname{dim}_{R}(A)$. In this paper, we use the terminology of Kirby (see [9]).

Lemma 2.2 ([1]). (i) $\mathrm{N}-\operatorname{dim}_{R}(A) \leqslant$ $\operatorname{dim}\left(R / \operatorname{Ann}_{R} A\right)$, and the equality holds if $A$ satisfies the property $\left({ }^{*}\right)$.

(ii) $\mathrm{N}-\operatorname{dim}_{R}\left(H_{\mathfrak{m}}^{i}(M)\right) \leq i$, for all $i$.

The following property of attached primes of the local cohomology under localization is known as Weak general Shifted Localization Principle (see [10]).

Lemma 2.3. We have $\operatorname{Att}_{R_{\mathfrak{p}}}\left(H_{\mathfrak{p} R_{\mathfrak{p}}}^{i-\operatorname{dim} R / \mathfrak{p}}\left(M_{\mathfrak{p}}\right)\right.$ is the subset of $\left\{\mathfrak{q} R_{\mathfrak{p}} \mid \mathfrak{q} \in\right.$ $\left.\min \operatorname{Att}_{R}\left(H_{\mathfrak{m}}^{i}(M)\right), \mathfrak{q} \subseteq \mathfrak{p}\right\}$, for all $\mathfrak{p} \in$ $\operatorname{Spec}(R)$.

For an integer $i \geq 0$, following M. Brodmann and R. Y. Sharp (see [11]), the $i$-th pseudo support of $M$, denoted by $\operatorname{Psupp}_{R}^{i}(M)$, is defined by the set

$$
\left\{\mathfrak{p} \in \operatorname{Spec} R \mid H_{\mathfrak{p} R_{\mathfrak{p}}}^{i-\operatorname{dim} R / \mathfrak{p}}\left(M_{\mathfrak{p}}\right) \neq 0\right\} .
$$

Note that the role of $\operatorname{Psupp}_{R}^{i}(M)$ for the Artinian $R$-module $A=H_{\mathfrak{m}}^{i}(M)$ is in some sense similar to that of $\operatorname{Supp} L$ for a finitely generated $R$-module $L$, cf. [11], [5]. Although, we always have $\operatorname{Supp} L=$ $\operatorname{Var}\left(\operatorname{Ann}_{R} L\right)$, but the analogous equality $\operatorname{Psupp}_{R}^{i}(M)=\operatorname{Var}\left(\operatorname{Ann}_{R} H_{\mathfrak{m}}^{i}(M)\right)$ is not valid in general. The following lemma gives a necessary and sufficient conditions for the above equality.

Lemma $2.4([5])$. Let $i \geq 0$ be an integer. Then the following statements are equivalent: (i) $H_{\mathfrak{m}}^{i}(M)$ satisfies the property $\left(^{*}\right)$.

(ii) $\operatorname{Var}\left(\operatorname{Ann}_{R}\left(H_{\mathfrak{m}}^{i}(M)\right)\right)=\operatorname{Psupp}_{R}^{i} M$.

In particular, if $H_{\mathfrak{m}}^{i}(M)$ satisfies the property $\left(^{*}\right)$ then

$$
\min \operatorname{Att}_{R}\left(H_{\mathfrak{m}}^{i}(M)\right)=\min \operatorname{Psupp}_{R}^{i} M .
$$

In 2010, N. T. Cuong, L. T. Nhan and N. T. K. Nga (see [12]) used pseudo support to describe the non-Cohen-Macaulay locus of $M$. Recall that $M$ is equidimensional if $\operatorname{dim}(R / \mathfrak{p})=d$, for all $\mathfrak{p} \in \min ($ Ass $M)$.

Lemma 2.5 ([12]). Suppose that $M$ is equidimensional and the ring $R / \operatorname{Ann}_{R} M$ is catenary. Then $\operatorname{Psupp}_{R}^{i}(M)$ is closed for $i=0,1, d$ and $\operatorname{nCM}(M)=\bigcup_{i=0}^{d-1} \operatorname{Psupp}_{R}^{i}(M)$, where $\operatorname{nCM}(M)$ is the Non Cohen-Macaulay )) locus of $M$.

Following M. Nagata ([3]), we say that $M$ is unmixed if $\operatorname{dim}(\widehat{\mathrm{R}} / \widehat{\mathfrak{p}})=d$ for all prime ideals $\widehat{\mathfrak{p}} \in$ Ass $\widehat{M}$, and $M$ is quasi unmixed if $\widehat{M}$ is equidimensional. The next lemma show that the property $(*)$ for the local cohomology modules $H_{\mathfrak{m}}^{i}(M)$ of levels $i<d$ is closed related to the universal catenaricity and unmixedness of certain local rings.

Lemma 2.6 ([5]). Assume that $H_{\mathfrak{m}}^{i}(M)$ satisfies the property $\left({ }^{*}\right)$ for all $i<d$. Then $R / \mathfrak{p}$ is unmixed for all $\mathfrak{p} \in$ Ass $M$ and the ring $R / \mathrm{Ann}_{R} M$ is universally catenary.

Proof of Theorem 1.2. It follows from the Lemma 2.6 that $R / \mathfrak{p}=R / \operatorname{Ann}_{R}(R / \mathfrak{p})$ is universally catenary.

Set $S$ to be the image of $R \backslash \mathfrak{p}$ in $\widehat{R}$. We have

$$
R_{\mathfrak{p}} / \mathfrak{p} R_{\mathfrak{p}} \otimes_{R} \widehat{R} \cong S^{-1}(\widehat{R} / \mathfrak{p} \widehat{R}) .
$$

We need to prove $\left(S^{-1}(\widehat{R} / \mathfrak{p} \widehat{R})\right)_{S^{-1} \widehat{\mathfrak{q}}}$ is Cohen-Macaulay for all $\widehat{\mathfrak{q}} \in \operatorname{Spec}(\widehat{R})$ such 
that $(\widehat{\mathfrak{q}} \cap R) \cap S=\emptyset$. Assume that the statement is not true. Since

$$
\left(S^{-1}(\widehat{R} / \mathfrak{p} \widehat{R})\right)_{S^{-1} \widehat{\mathfrak{q}}} \cong(\widehat{R} / \mathfrak{p} \widehat{R})_{\widehat{\mathfrak{q}}}
$$

as $\widehat{R}_{\widehat{\mathfrak{q}}}$-module, there exists $\widehat{\mathfrak{q}} \in \operatorname{Spec}(\widehat{R}), \widehat{\mathfrak{q}} \cap$ $S=\emptyset$ such that $(\widehat{R} / \mathfrak{p} \widehat{R})_{\widehat{\mathfrak{q}}}$ is not Cohen-Macaulay. Then there exists $\widehat{\mathfrak{p}} \in$ $\operatorname{Spec}(R), \widehat{\mathfrak{q}} \supseteq \widehat{\mathfrak{p}},(\widehat{\mathfrak{p}} \cap R) \cap S=\emptyset$ and $\widehat{\mathfrak{p}} \in$ $\operatorname{Min} \operatorname{nCM}(\widehat{R} / \widehat{\mathfrak{p}} \widehat{R})$. Hence,

$$
\operatorname{nCM}\left((\widehat{R} / \widehat{\mathfrak{p}} \widehat{R})_{\widehat{\mathfrak{p}}}\right)=\left\{\widehat{\mathfrak{p}} \widehat{R}_{\widehat{\mathfrak{p}}}\right\} .
$$

We have $R / \mathfrak{p}$ is unmixed by Lemma 2.6. So $\widehat{R} / \widehat{\mathfrak{p}} \widehat{R}$ is equidimensional. Hence $(\widehat{R} / \widehat{\mathfrak{p}} \widehat{R})_{\widehat{\mathfrak{p}}}$ is equidimensional. On the other hand, since $(\widehat{R} / \widehat{\mathfrak{p}} \widehat{R})_{\widehat{\mathfrak{p}}}$ is the image of a Cohen-Macaulay ring, $(\widehat{R} / \widehat{\mathfrak{p}} \widehat{R})_{\widehat{p}}$ is generalized Cohen-Macaulay.

Set $s=\operatorname{dim} \widehat{R} / \widehat{\mathfrak{p}} \widehat{R}=\operatorname{ht}(\widehat{\mathfrak{p}} / \mathfrak{p} \widehat{R})$. By Lemma 2.5 , we have

$$
\operatorname{nCM}(\widehat{R} / \widehat{\mathfrak{p}} \widehat{R})_{\widehat{\mathfrak{p}}}=\bigcup_{i=0}^{s-1} \operatorname{Psupp}_{\widehat{R}}^{i}\left((\widehat{R} / \widehat{\mathfrak{p}} \widehat{R})_{\widehat{\mathfrak{p}}}\right) .
$$

Therefore, there exists $i<s$ such that $H_{\widehat{\mathfrak{p}} \widehat{R}_{\widehat{\mathfrak{p}}}}^{i}(\widehat{R} / \mathfrak{p} \widehat{R})_{\widehat{p}} \neq 0$. On the other hand,

$$
\ell\left(H_{\widehat{\mathfrak{p}} \widehat{R}_{\widehat{p}}}^{i}(\widehat{R} / \mathfrak{p} \widehat{R})_{\widehat{p}}\right)<\infty .
$$

Then

$$
\operatorname{Att}_{\widehat{R}}\left(H_{\widehat{p} \widehat{R}_{\widehat{p}}}^{i}(\widehat{R} / \mathfrak{p} \widehat{R})_{\widehat{p}}\right)=\left\{\mathfrak{p} \widehat{R}_{\widehat{p}}\right\} .
$$

It is followed by Weak general Shifted Localization Principle (Lemma 2.3) that $\widehat{\mathfrak{p}} \in$ $\operatorname{Att}_{\widehat{R}}\left(H_{\mathfrak{m}}^{i+\operatorname{dim} \widehat{R} / \hat{\mathfrak{p}}}(\widehat{R} / \mathfrak{p} \widehat{R})\right)$. Set $j=i+$ $\operatorname{dim} \widehat{R} / \widehat{\mathfrak{p}}$. We have

$$
\begin{aligned}
j<\operatorname{ht} \widehat{\mathfrak{p}} / \mathfrak{p} \widehat{R}+\operatorname{dim} \widehat{R} / \widehat{\mathfrak{p}} & \leq \operatorname{dim} \widehat{R} / \mathfrak{p} \widehat{R} \\
& =\operatorname{dim} R / \mathfrak{p} .
\end{aligned}
$$

Hence, $\mathfrak{p} \in \operatorname{Att}_{R}\left(H_{\mathfrak{m}}^{j}(R / \mathfrak{p})\right)$ by Lemma 2.1. By Lemma 2.2

$$
\begin{aligned}
\mathrm{N}-\operatorname{dim} H_{\mathfrak{m}}^{j}(R / \mathfrak{p}) \leq j & <\operatorname{dim} R / \mathfrak{p} \\
& \leq R / \operatorname{Ann}_{R} H_{\mathfrak{m}}^{j}(R / \mathfrak{p}) .
\end{aligned}
$$

This implies that $H_{\mathfrak{m}}^{j}(R / \mathfrak{p})$ does not satisfy the property $(*)$. It is in contradiction to the hypothesis. Therefore, all its formal fibers over $\mathfrak{p}$ are Cohen-Macaulay.

\section{Conclusion}

The paper gives a relation between the property $\left(^{*}\right)$ of local cohomology module and structure of base ring. In detail, we prove that for each $\mathfrak{p} \in \operatorname{Spec}(R)$ such that $H_{\mathfrak{m}}^{i}(R / \mathfrak{p})$ satisfies the property $\left(^{*}\right)$ for all $i$, then $R / \mathfrak{p}$ is universally catenary and the formal fibre of $R$ over $\mathfrak{p}$ is Cohen-Macaulay.

\section{References}

[1]. C. T. Nguyen and N. T. Le, "On the Noetherian dimension of Artinian modules," Vietnam Journal of Mathematics, vol. 30, no. 2, pp. 121-130, 2002.

[2]. D. Ferrand and M. Raynaud, "Fibres formelles d'un anneau local Noetherian," Annales Scientifiques de l'École Normale Supérieure, vol. 3, no. 4, pp. 295-311, 1970.

[3]. M. Nagata, Local rings, Interscience, New York, 1962.

[4]. C. T. Nguyen, D. T. Nguyen and N. T. Le, "Top local cohomology and the catenaricity of the unmixed support of a finitely generated module," Communications in Algebra, vol. 35, no. 5, pp. 1691-1701, 2007.

[5]. N. T. Le and A. N. Tran, "On the unmixedness and the universal catenaricity of local rings and local cohomology modules," Journal of Algebra, vol. 321, pp. 303-311, 2009 . 
[6]. N. T. Le and C. D. M. Tran, "Noetherian dimension and co-localization of Artinian modules over local rings," $A l$ gebra Colloquium, vol. 21, pp. 663-670, 2014.

[7]. I. G. Macdonald, "Secondary representation of modules over a commutative ring," Symposia Mathematica, vol. 11 , pp. 23-43, 1973.

[8]. R. N. Roberts, "Krull dimension for Artinian modules over quasi local commutative rings," Quarterly Journal of Mathematics, vol. 26, no. 2, pp. 269273, 1975.

[9]. D. Kirby, "Dimension and length of Artinian modules," Quarterly Journal of
Mathematics, vol. 41, no. 2, pp. 419429, 1990.

[10]. M. Brodmann and R. Y. Sharp, Local cohomology: an algebraic introduction with geometric applications, Cambridge University Press, 1998.

[11]. M. Brodmann and R. Y. Sharp, "On the dimension and multiplicity of local cohomology modules," Nagoya Mathematical Journal, vol. 167, pp. 217-233, 2002.

[12]. C. T. Nguyen, N. T. Le and N. K. T. Nguyen, "On pseudo supports and non-Cohen-Macaulay locus of finitely generated modules," Journal of Algebra, vol. 323, pp. 3029-3038, 2010. 